\title{
Virtual Biopsy and Physical Characterization of Tissues, Biofilms, Implants and Viscoelastic Liquids Using Vibrational Optical Coherence Tomography
}

\author{
Frederick H. Silver ${ }^{1}{ }^{*}$, Ruchit G. Shah ${ }^{2}$, Dominick Benedetto ${ }^{3}$, Abhinav Dulur ${ }^{2}$, Thomas Kirn' ${ }^{1}$ \\ ${ }^{1}$ Department of Pathology and Laboratory Medicine, Robert Wood Johnson Medical School, Rutgers, The State University of New \\ Jersey, Piscataway, NJ, USA \\ ${ }^{2}$ Graduate Program in Biomedical Engineering, Rutgers, The State University of New Jersey, Piscataway, NJ, USA \\ ${ }^{3}$ Center for Advanced Eye Care, Vero Beach, FL, USA \\ Email: *silverfr@rutgers.edu, ${ }^{*}$ fhsilver@hotmail.com
}

How to cite this paper: Silver, F.H., Shah, R.G., Benedetto, D., Dulur, A. and Kirn, T. (2019) Virtual Biopsy and Physical Characterization of Tissues, Biofilms, Implants and Viscoelastic Liquids Using Vibrational Optical Coherence Tomography. World Journal of Mechanics, 9, 1-16.

https://doi.org/10.4236/wjm.2019.91001

Received: January 10, 2019

Accepted: January 28, 2019

Published: January 31, 2019

Copyright $\odot 2019$ by author(s) and Scientific Research Publishing Inc. This work is licensed under the Creative Commons Attribution International License (CC BY 4.0).

http://creativecommons.org/licenses/by/4.0/

\begin{abstract}
Tissue biopsies and implant analysis during animal testing or clinical studies are a requirement for development of new surgical materials and procedures. In this paper we report the use of vibrational OCT (VOCT) to evaluate the viscoelastic behavior of tissues, polymeric materials, biofilms, and viscoelastic solutions of macromolecules. Our results suggest that VOCT is a useful technique to characterize the behavior of cellular tissues and biofilms, polymeric implant materials and viscoelastic solutions used in medicine. It is demonstrated that the modulus and resonant frequency squared per unit thickness is a feature that can be used to characterize a variety of tissues. Further work is needed to understand the generalized behavior of synthetic polymers and viscoelastic solutions.
\end{abstract}

\section{Keywords}

Optical Coherence Tomography, Vibrational Optical Coherence

Tomography, Biofilm, Viscoelastic Solutions, Mechanical Properties,

Modulus, Stiffness, Tissues, Hyaluronic Acid

\section{Introduction}

Tissue biopsies and implant analysis during animal testing or clinical studies are a requirement for development of new surgical materials and procedures. The need to minimize the number of animals used in pre-clinical testing of medical 
devices and the ethical challenge of doing surgical biopsies on patients with skin lesions has provided the impetus to develop non-invasive imaging methods such as magnetic resonance (MRI), ultrasound and optical coherence tomography (OCT) [1] techniques to study tissues and implants. However, since images alone cannot give a complete "picture" of the nature of the tissue or implant, we have developed a technique termed vibrational OCT (VOCT) [2] [3] [4] [5]. This technique allows researchers to non-invasively and non-destructively measure the mechanical properties of tissues and implants and provides an image that can be used to evaluate biological processes that occur in tissues and at tissue-implant interfaces in vivo. In addition, this technique can be used to non-invasively and non-destructively evaluate the mechanical properties of implants in vitro [3].

VOCT has potential applications in evaluating the progression of healing of surface wounds, the margins of skin and surface lesions, the efficacy of cosmetic and reconstructive procedures, biofilm formation and removal, and implant mechanical biodegradation. OCT is a technique used extensively in Ophthalmology and is gaining acceptance in Dermatology and Gastroenterology.

OCT is a non-invasive, non-destructive optical technique for imaging tissue. It has a penetration depth of between 0.5 and $2 \mathrm{~mm}$ depending on the light source used and the manner in which the reflected light is analyzed. It has been used to: detect and diagnose non-melanoma skin cancer [6], visualize the functional microvasculature of the skin [7], evaluate the oxidative effects of hair dying [8], determine the margins of basal cell carcinomas before micrographic surgery [9], and map vascularization in plaque psoriasis [10]. Quantitative uses of OCT include measurement of the surface distance to the first vessels [11], characterization of cutaneous wounds [12], thickness measurements in basal cell carcinoma and malignant melanoma [13], differentiation of benign and malignant melanoma [13] [14], and OCT capillaroscopy of nail folds [15]. Besides evaluation of skin lesion pathology, OCT has potential uses in following biofilm formation in skin wounds, biofilm associated contamination of implants and characterization of viscoelastic liquids used in surgery.

A biofilm is the accumulation of microorganisms and their extracellular products to form a highly structured bacterial community on a surface of tissue or an implant. Biofilms are present on epithelium and mucous membranes of human tissues, at insertion sites for medical devices, and form from airborne contamination [16]. Their presence leads to medical conditions such as prosthetic value endocarditis, keratitis due to contact lens contamination, bacteremia in urinary catheters, intravascular catheter infections, septic loosening of joint prostheses, prolonged skin wound healing, periodontal disease and caries, ventilator associated pneumonia, lower respiratory infections, and surgical site infections [17]. Pseudomonas aeruginosa has been reported to be found in skin wounds and infected leg ulcers. Staphylococcus epidermidis and Staphylococcus aureus have been associated with biofilm formation on medical devices [17]. There are no methods available for rapid detection of biofilm formation in a clinical setting [17]. 
In this paper we report the use of VOCT to evaluate the viscoelastic behavior of tissues, polymeric materials, biofilms, and viscoelastic solutions of macromolecules. Our results suggest that VOCT is a useful technique to characterize the behavior of cellular tissues and biofilms, polymeric implant materials and viscoelastic solutions used ophthalmic and orthopedic surgery.

\section{Materials and Methods}

\subsection{Materials}

\subsubsection{Calibration Materials}

Human decellularized dermal samples, approximately $1.0 \mathrm{~cm}$ wide, $5.0 \mathrm{~cm}$ long and $1.0 \mathrm{~mm}$ thick, were obtained from allograft tissue as described previously [2] [3] [4] [5]. Decellularized human dermal samples were tested after immersion in phosphate buffer solution as described elsewhere [2] [3] [4] [5]. All samples were tested wet after soaking in phosphate buffer solution at $\mathrm{pH} 7.4$ for at least 30 minutes. Processing and testing steps were conducted at $22^{\circ} \mathrm{C}$.

\subsubsection{Tissue}

Porcine rib fat, approximately $2 \mathrm{~mm}$ in thickness, was obtained from Spear Products (Coopersburg, $\mathrm{Pa}$ ) in strips approximately $5 \mathrm{~mm}$ wide and 5 to $7 \mathrm{~cm}$ in length. The fat was stored frozen at $-6^{\circ} \mathrm{F}$ until it was tested.

\subsubsection{Biofilm Preparation}

S. aureus biofilms were prepared from stock frozen solutions from the microbiology lab at Robert Wood Johnson University Hospital. The frozen $S$. aureus stock was inoculated to blood agar plates and incubated at $37^{\circ} \mathrm{C}$ for $24 \mathrm{hrs}$. Five milliliters of Gibco LB broth was placed in a cell culture plate. Using a sterile wooden applicator stick, $S$. aureus colonies were inoculated onto the cell culture dish by picking a colony and introducing it onto the cell culture plate. The bacteria were incubated for 48 hours at $37^{\circ} \mathrm{C}$ without agitation. After incubation, the cell culture plate was removed from the incubator and the LB medium was aspirated using a p-1000 pipette. The biofilm was present on the bottom of the cell culture plate and the sample was tested in the tissue culture plate.

Commercial samples of Provisc and Viscoat viscoelastic solutions were obtained from Alcon, a Novatis company, and used as received. A drop containing between 0.2 and $0.5 \mathrm{ml}$ of each sample was placed on a glass slide for examination.

New Viton rubber gaskets were obtained from Blaylock Gasket and Packing as well as a used gasket was obtained from a Ford transmission with approximately 100,000 miles on the odometer.

\section{Methods}

\subsection{Imaging}

Images of the samples were made by photographing the samples using a Samsung S8 cell phone. OCT images were made using a Lumedica OQ Labscope 
(Lumedica, Inc., Durham, NC) operating in the scanning mode as described previously [4] [5].

\subsection{Mechanical Testing}

Incremental stress-strain measurements In Vitro.

Samples were tested in uniaxial tension at $22^{\circ} \mathrm{C}$ by adding a strain increment and then measuring the load before an additional strain step was added as described previously [2] [3] [4] [5]. Strain rates of approximately $30 \%$ per minute were used in the tensile experiments. Varying axial deformations of between 1 and about $11 \%$ were applied through adjustment of a graduated translation stage. The resulting axial force $(F)$ was measured by the force gage and recorded for subsequent calculations. Stress values were calculated from the experimentally measured force divided by the cross-sectional area. Strains were calculated by dividing the change in length by the original length based on the movement of the translational stage after each strain increment was added. The tensile modulus was calculated from a tangent drawn to the stress-strain curve at the strain increment used as described previously [2] [3] [4] [5].

\subsection{Measurement of the Resonant Frequency}

Samples were tested using vibrational $\mathrm{OCT}$ at $22^{\circ} \mathrm{C}$ by applying a sinusoidal sound wave from a speaker placed beneath the sample as discussed previously [2] [3] [4] [5]. Calibration samples and polymer strips were placed in tension by clamping the samples at the ends using grips connected to a moveable stage. The strain applied was determined from the movement of a micrometer attached to the stage. One of the grips was attached to a force gage.

Tissue culture plates were placed on a rigid hollow frame above the speaker and vibrated from below. Drops of viscoelastic ophthalmic solutions were placed on a glass slide and placed on the rigid frame above the speaker. The frame, speaker, tissue culture dish and glass slide resonant frequencies were measured in the absence of the samples. Sample weighted displacements were corrected for any resonant frequencies of the speaker and support materials or from line voltage variations.

To calculate the vibrational modulus, transverse forces were applied to the sample by positioning an acoustic loudspeaker (Intervox S225RA-40) beneath the sample. A function generator (Agilent) was used to drive the speaker with sinusoidal waveforms at varying amplitude and frequency. The resonant frequency was determined as the frequency at which the maximum displacement was observed as described previously [2] [3] [4] [5].

Transverse sample displacement was measured by spectral-domain optical coherence tomography (SD-OCT), a non-contact, interferometric technique as discussed previously [2] [3] [4] [5]. The resonant frequency of each sample was initially estimated at a single point by measuring the transverse displacement resulting from sinusoidal driving frequencies ranging from $30 \mathrm{~Hz}$ to $700 \mathrm{~Hz}$, in steps of $50 \mathrm{~Hz}$. Once the region where the maximum frequency was identified, 
smaller steps of $10 \mathrm{~Hz}$ were used to more accurately identify the peak frequency and the actual resonant frequency, $f_{n}$.

$$
E=m\left(2 \pi f_{n}\right)^{2}\left(\frac{L}{A}\right)
$$

The modulus from in vitro vibrational studies on calibration materials, fat, biofilms and rubber gaskets were determined using Equation (1) where $m, L$ and $A$ are the sample mass, length and cross-sectional area [2] [3] [4] [5]. The biofilm thickness was estimated by weighing the Petri dish before and after the biofilm was grown on the dish and calculating the thickness knowing the culture dish area and assuming a density of 1.0. The mass and volume of drops of viscoelastic solutions tested were used to calculate the moduli of viscoelastic materials studied.

\subsection{Measurement of Elastic and Viscous Behaviors}

The elastic and viscous components of the viscoelastic behavior were obtained from measurements made from the driving frequency peak as described previously. The elastic component was obtained from the peak height while the viscous component was obtained by dividing the change in frequency at the half height of the peak (i.e. $3 \mathrm{db}$ down from maximum peak in power spectrum) by the driving frequency. This method is known as the half-height bandwidth method discussed by Paul Macioce

(https://www.roush.com/wp-content/uploads/2015/09/Insight.pdf) [18].

\section{Results}

A list of the samples tested and their resonant frequencies are shown in Table 1. Photographic images of new and old Viton rubber samples and the Staph culture are shown in Figure 1. The OCT images of the rubber samples, biofilms and the viscoelastic solutions did not reveal any significant details and are not shown due to the difficulty with getting clear images of these materials. An OCT image of fat is shown in Figure 2 and reveals details at the level of groups of cells. In the fat OCT image groups of cells (dark spots) are seen in juxtaposition to neighboring extracellular matrix.

Plots of weighted displacement versus frequency for $S$ aureus biofilm (top), new Viton rubber gasket (middle) and old Viton rubber gasket (bottom) are shown in Figure 3. Note the low value of the resonant frequency for cellular materials in the biofilm samples compared to polymeric samples. Also note the reduction of the resonant frequency in the old Viton sample compared to the new gasket. The tensile modulus of the new Viton gasket was $12.27 \mathrm{MPa}$ and was reduced to $5.81 \mathrm{MPa}$ in the old gasket (see Table 1). The tensile stress-strain curves for new (top) and old (bottom) Viton rubber gaskets are shown in Figure 4 and indicate that the old gasket required less stress to stretch to a fixed strain as compared to the new one.

Weighted displacement versus frequency for Provisc (top), Viscoat (middle) and water droplets on a glass slide (bottom) determined from vibrational OCT 
Table 1. Samples tested using vibrational optical coherence tomography.

\begin{tabular}{ccccc}
\hline Sample & Description & Resonant Frequency (Hz) & \multicolumn{2}{c}{ Modulus (MPa) } \\
\cline { 4 - 5 } & & & Tensile & Vibrational \\
\hline Decellularized Dermis & Calibration Standard & 150 & $2.69+/-0.021$ & $2.46+/-0.01$ \\
Porcine Fat & Cellular Model & $30+/-5$ & NA & $0.03+/-0.01$ \\
Staphylococcus Culture & Biofilm Model & $40+/-5$ & NA & $0.056+/-0.014$ \\
Provisc & Viscoelastic Solution & 90,140 & NA & $7.99 \mathrm{E}-3+/-0.019$ \\
Viscoat & Viscoelastic Solution & 100,150 & NA & $9.86 \mathrm{E}-3+/-0.022$ \\
Water & Model & 60 & NA & $3.55 \mathrm{E}-3$ \\
Viton Rubber New & Implant Model & $180+/-5$ & $12.27+/-0.19$ & $11.45+/-0.64$ \\
Viton Rubber Old & Implant Model & $140+/-5$ & $5.81+/-0.16$ & $5.99+/-0.43$ \\
\hline
\end{tabular}

${ }^{\star}$ Sample strain $5 \%$.

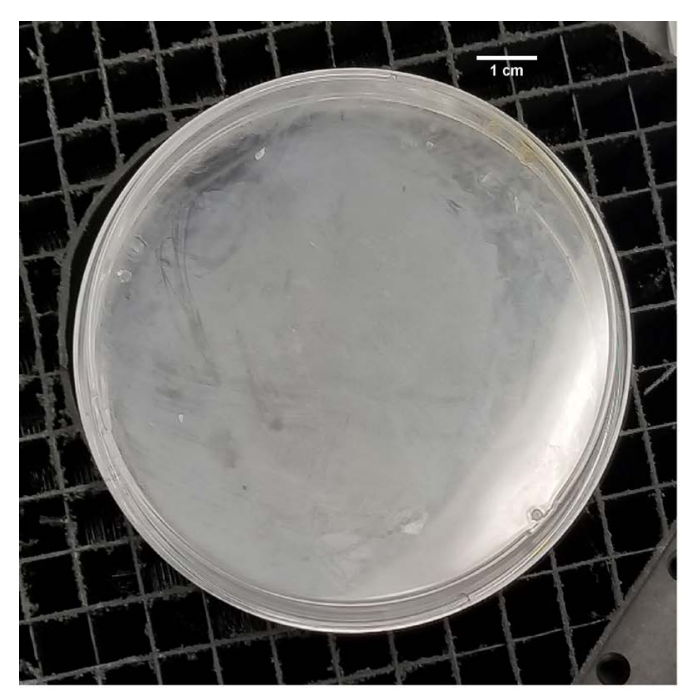

(a)

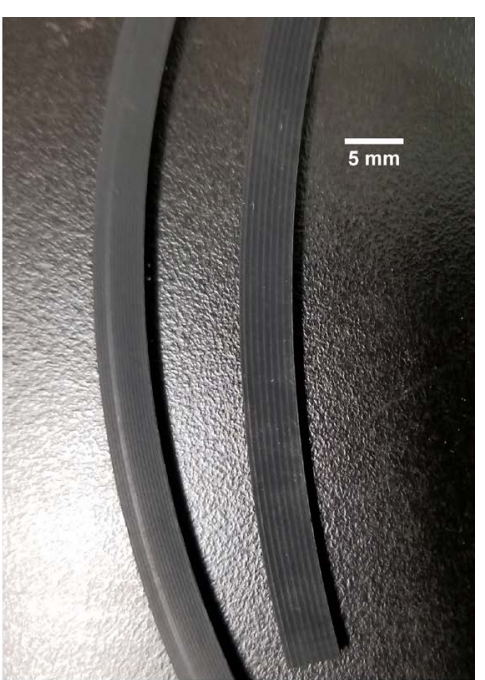

(b)

Figure 1. Photographic images of Staphylococcus aureus biofilm (top) and Viton rubber new (left) and worn (right) gaskets.

are shown in Figure 5. The water droplet peak as a function of frequency was subtracted from the peaks to obtain the data for Provisc and Viscoat shown in Figure 5. All peaks are listed in Table 1.

The modulus as a function of frequency for new (top) and old (bottom) Viton rubber gaskets is shown in Figure 6. The modulus measured is maximized at the resonant frequency while the loss modulus in percent of the total modulus as a function of frequency for new (top) and old (bottom) is shown in Figure 7. The loss \% decreases more rapidly with increasing frequency for the old Viton rubber gasket.

In Figure 8 is shown a plot of modulus determined from vibrational OCT versus resonant frequency squared divided by the thickness determined from OCT. This plot illustrates that the elastic modulus of cellular materials and 


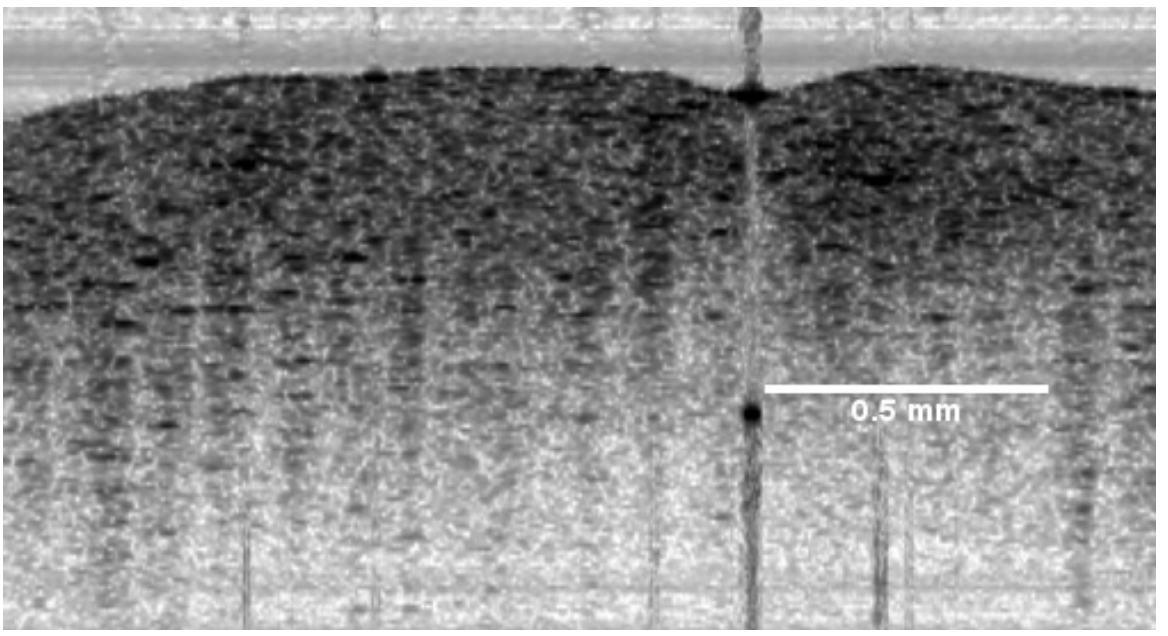

Figure 2. OCT images of porcine fat (top) observed with a Lumedica OQ Labscope operating in the scanning mode.

tissues can be used to determine the physical state of cellular extracellular matrix components non-invasively and non-destructively from vibrational OCT studies. This is accomplished by measuring the resonant frequency squared and dividing by the thickness of a material. Data for synthetic polymeric samples does not fit this plot.

\section{Discussion}

The ability to identify and characterize a material used in surgery both prior to and after implantation is an important aspect of materials testing in animal experimentation. This helps limit the number of animals used during pre-clinical studies as well as facilitates outcome analysis during pre-clinical and clinical trials. The ability to do a "virtual biopsy" during animal studies and clinical trials would provide additional information not currently available. The current inability to image and measure the physical properties of implants leads to increased animal euthanasia in preclinical studies as well as limitations in analyzing clinical study results required to understand the outcomes obtained with implants and regenerative technologies. It is not possible from histological studies alone to evaluate the residual mechanical properties of implants which are important criteria for evaluation of biodegradation rates.

We have developed a technique to image and measure the mechanical properties of tissues and implants using VOCT [2] [3] [4] [5]. This technique involves collecting images from OCT and then applying a sinusoidal sound wave to evaluate the resonant frequency of the material. The data generated includes a cross-sectional image and the displacement of the sample. If the frequency is varied from about $30 \mathrm{~Hz}$ to $700 \mathrm{~Hz}$, the resonant frequency can be calculated from the frequency at which the maximum displacement occurs. Once the resonant frequency is known the modulus can be calculated knowing the OCT sample thickness and using a calibration curve developed from measurements on tissues and polymers. 

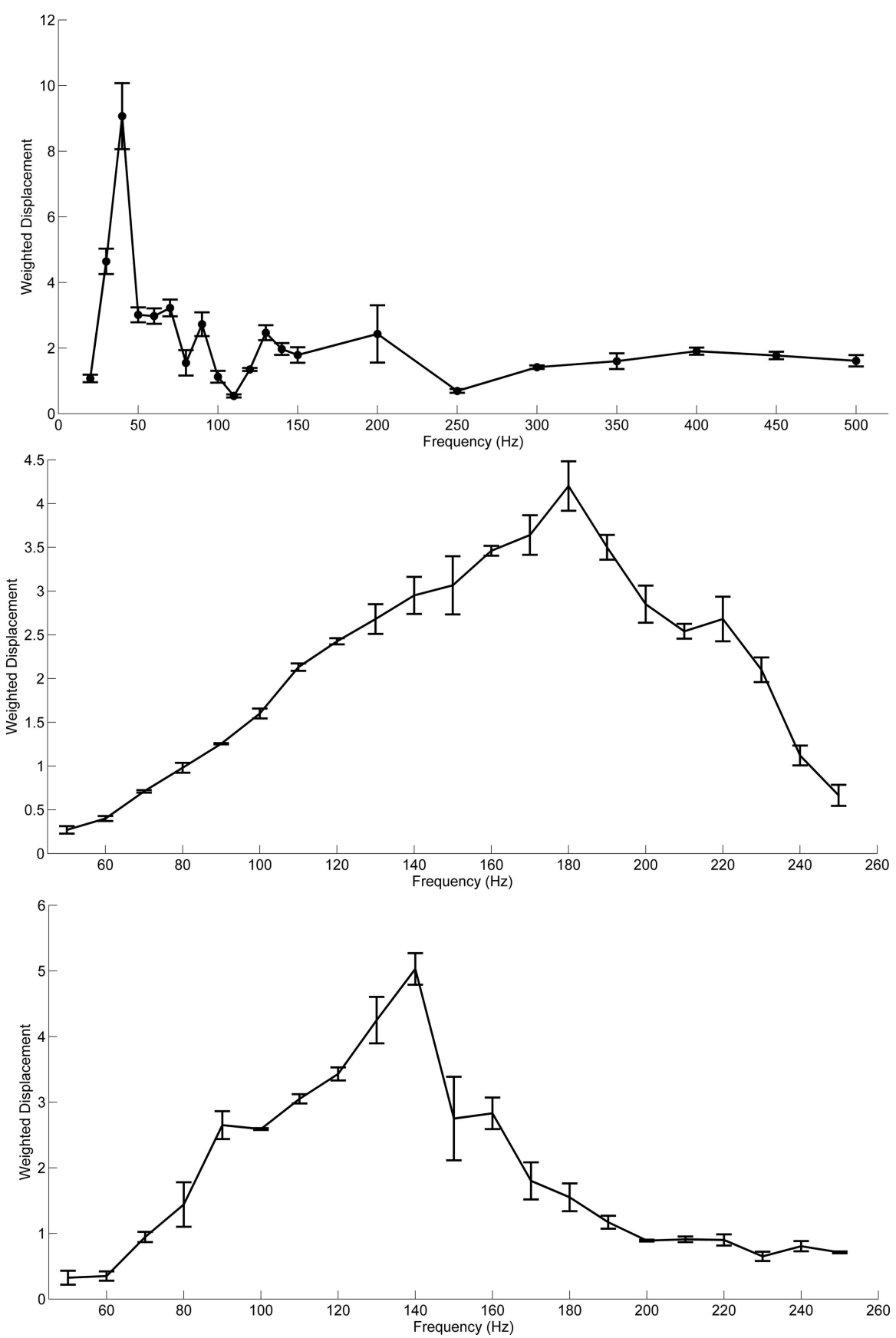

Figure 3. Weighted displacement versus frequency for Staphylococcus aureus biofilm (top), new Viton rubber gasket (middle) and old Viton rubber gasket (bottom). The resonant frequency is obtained from the frequency at which the maximum displacement is observed. The modulus can be obtained using Equation (1) from physical measurements made on the sample.

\subsection{Tissues}

Recently we have published OCT images and moduli values of human skin, scar, cornea and sclera [2] [3] [4] [5]. The data in these publications were obtained from plots of weighted displacement versus frequency measured from VOCT [2] [3] [4] [5] and were used to construct a calibration curve of modulus versus 

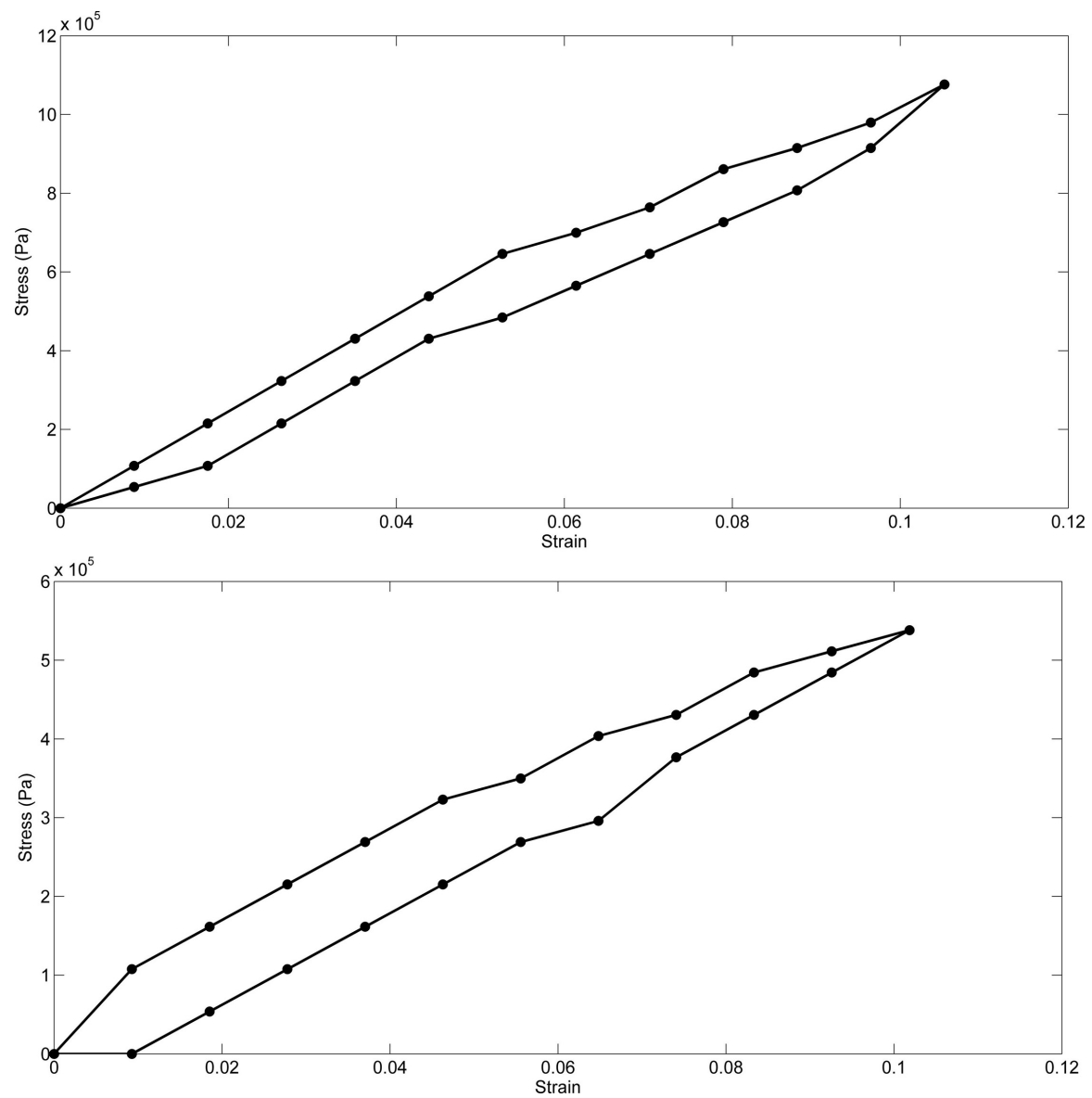

Figure 4. Tensile stress-strain curves for new (top) and old (bottom) Viton rubber gaskets. These curves were constructed using incremental tensile stress-strain measurements.

resonant frequency divided by sample thickness [5] for tissues and collagen samples. In this paper we extend those observations and report an approximate linear relationship exists between modulus and resonant frequency squared divided by the sample thickness for biological materials (see Figure 8). Using this relationship it is possible to identify tissue components seen in OCT images of biological materials based on their mechanical properties. It appears that the cellular materials are found at low values of the modulus in Figure 8 while the collagen-based materials are found at higher modulus values. Further studies will provide more data to evaluate whether this relationship can be applied to synthetic polymers; however the values for polymers studied in this study do not fall on the line in Figure 8.

\subsection{Polymers}

While the bulk of polymers used in medicine include, glycolic acid, polylactic acid, polydimethylsiloxane, polytetrafluoroethylene, polyethylene, polyacrylates, polyurethanes, and natural polymers such as collagen, hyaluronan, alginates and silk [1] [19], their properties vary extensively depending on how they are 

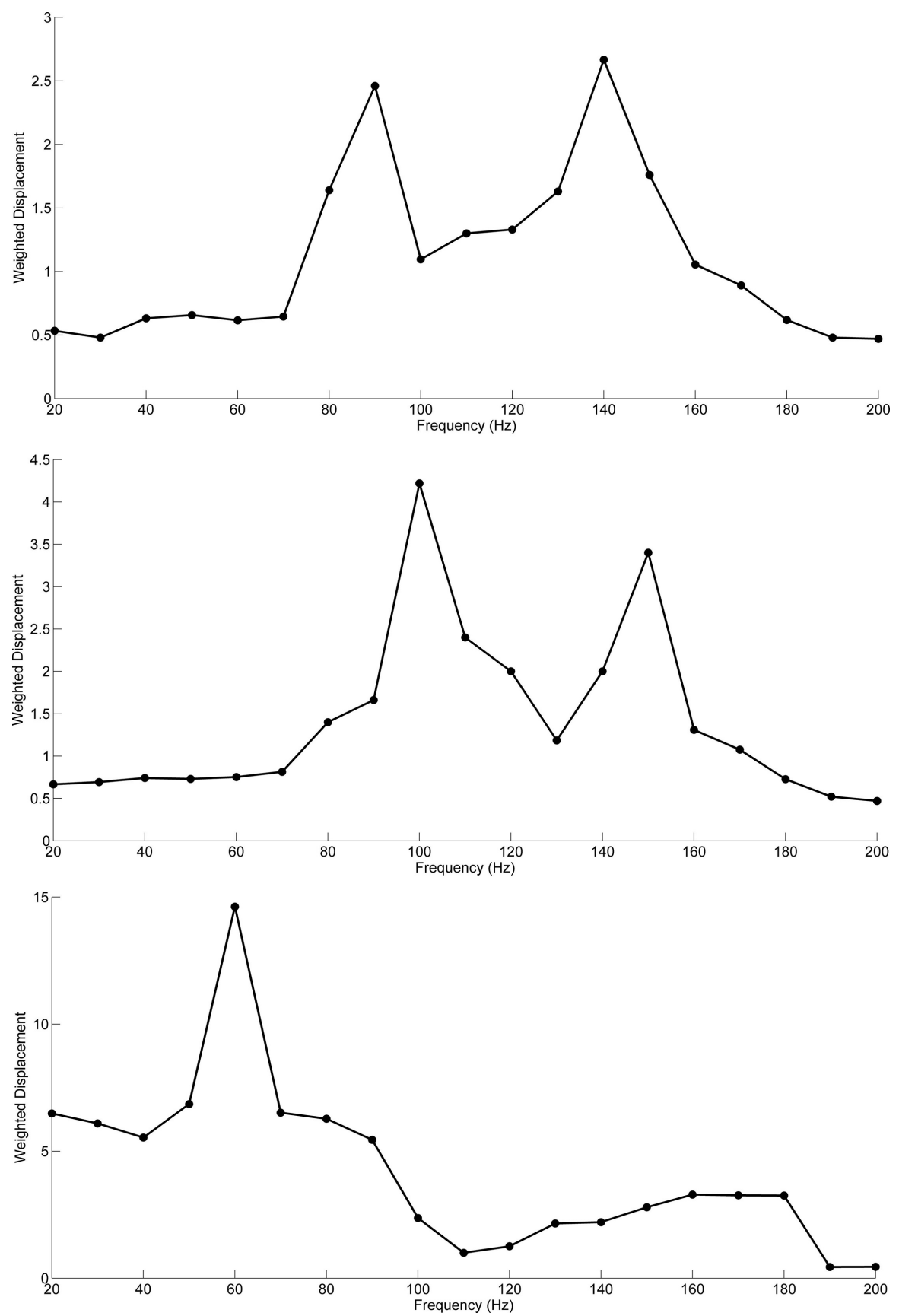

Figure 5. Weighted displacement versus frequency for Provisc (top), Viscoat (middle) and water droplets on a glass slide determined from vibrational OCT. The peak for water was subtracted from the peaks shown for Provisc and Viscoat, respectively.

compounded and processed. Numerous tests have been used to elucidate mechanical properties of tissues and implants including tensile, compressive, shear, hydrostatic compression, and three-point bending in one or more axial directions [19]. The majority of these tests are destructive in nature rendering the material useless after testing.

Polymers can be tested non-invasively and non-destructively using VOCT to show how processing steps affect the mechanical properties of the device. It is 

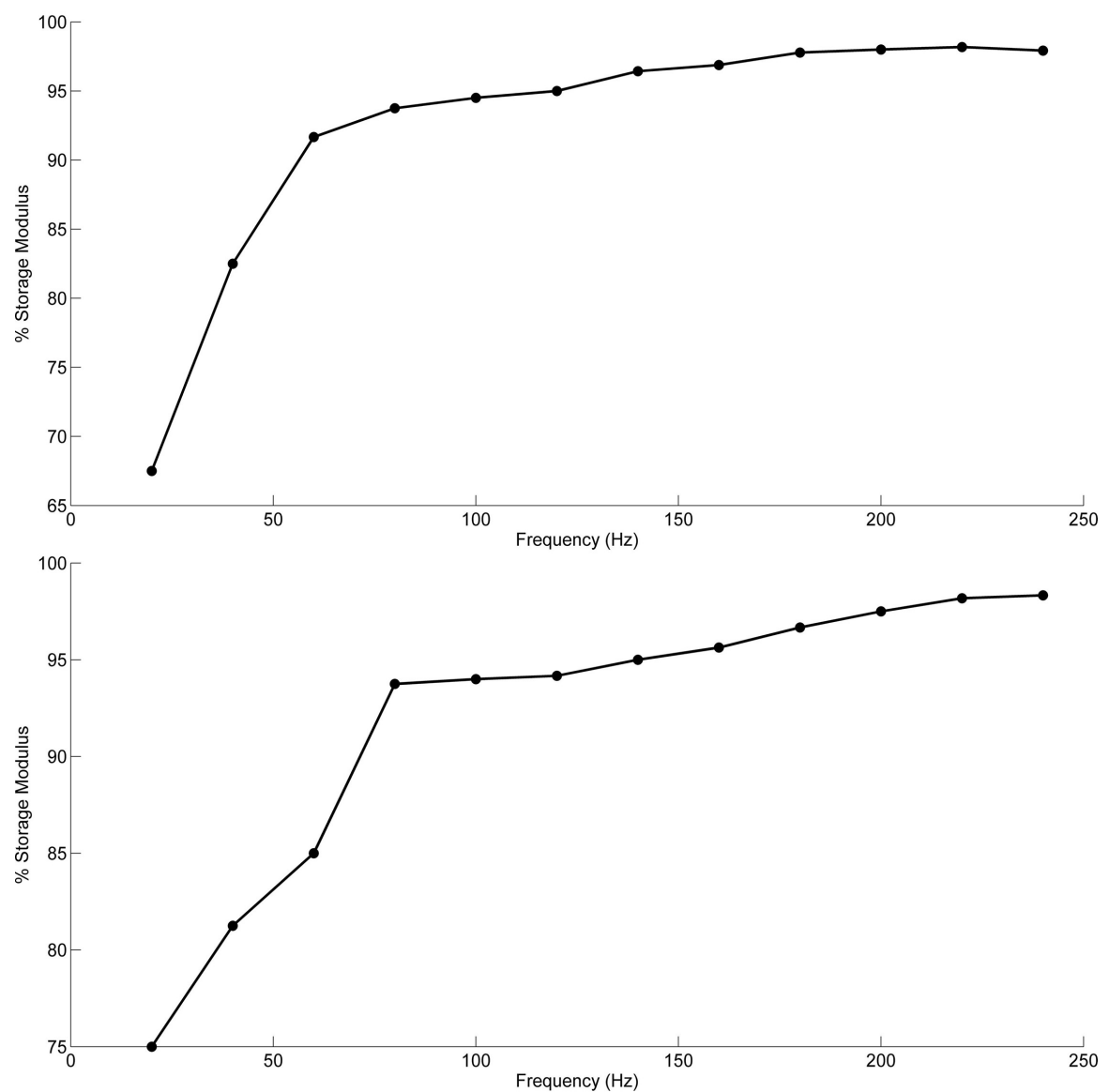

Figure 6. \% storage modulus as a function of frequency for new (top) and old (bottom) Viton rubber gaskets.

also possible to evaluate the effects of aging using VOCT. By cycling the sample at high frequencies it may possible to speed up aging analysis and evaluation of the effects of mechanical cycling.

In this study we report significant differences in the mechanical behavior of new and old Viton rubber gaskets. The old gasket after aging showed a reduced modulus. This led to a slightly higher viscous loss and ultimately would lead to premature mechanical failure. Using VOCT, polymeric implants can be evaluated for changes in mechanical properties during and after each processing step which will facilitate product development and shorten pre-clinical evaluation times.

\subsection{Biofilms}

A variety of bacteria have been reported to be present on biofilms found on tissues and implants including Staphylococcus epidermis, Pseudomonas aeruginosa, Staphylococcus aureus, and Escherichia coli (E. coli) [20] [21]. Layered S. epidermidis has been reported to be 1000 times more resistant to antibiotics compared to free floating cells [22]. Protective changes occur in bacteria after conversion from isolated cells to film-protected cells. 

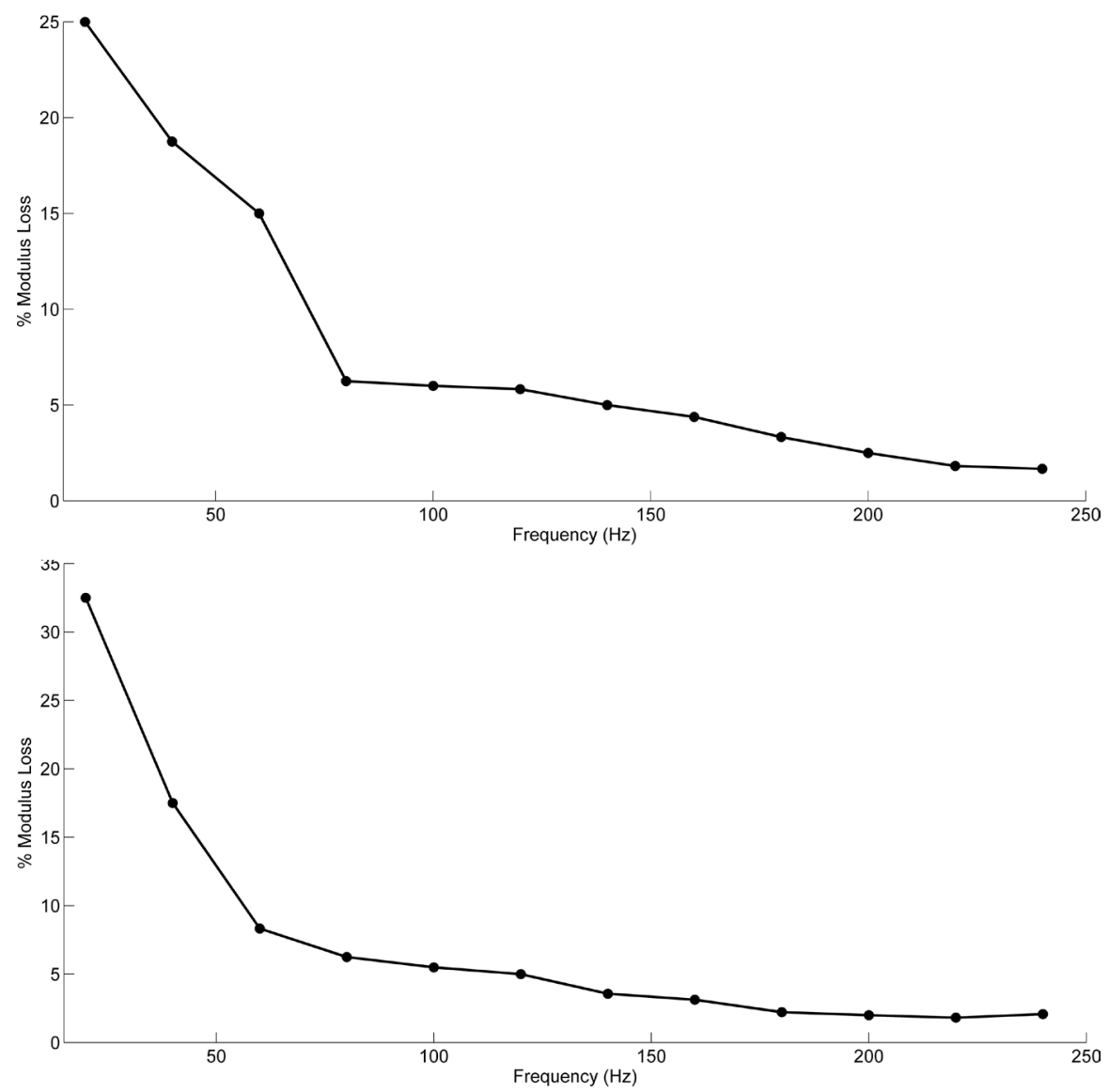

Figure 7. \% loss modulus as a function of frequency for new (top) and old (bottom) Viton rubber gasket.

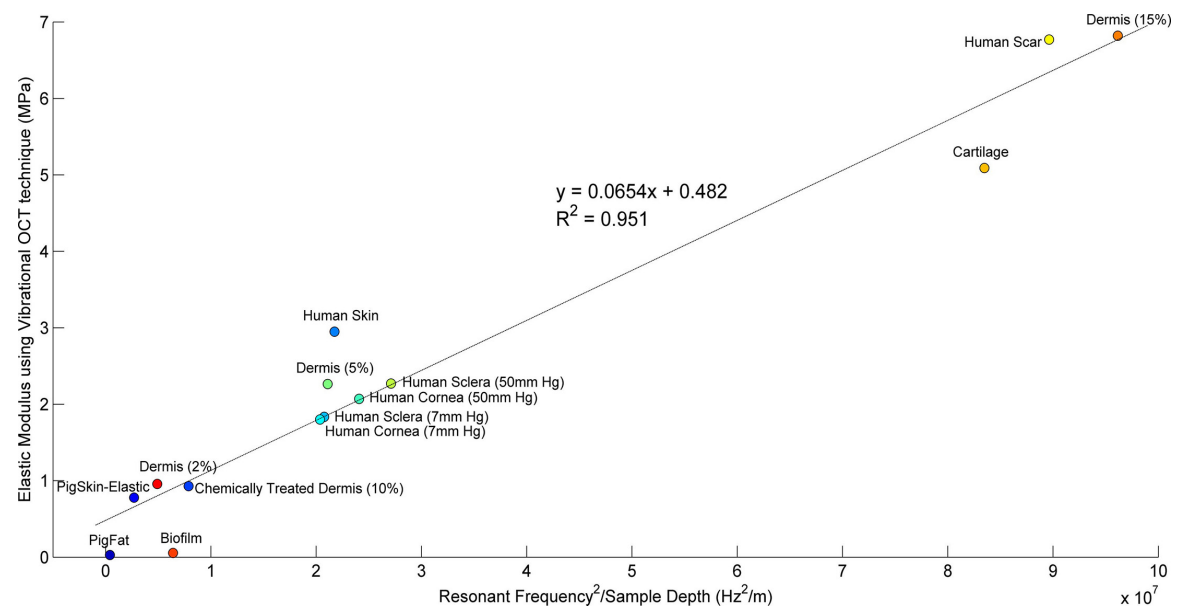

Figure 8. Plot of modulus determined from vibrational OCT versus resonant frequency squared divided by the thickness determined from OCT.

Biofilm formation steps include attachment, accumulation, maturation and detachment. There are specific genes that produce an adhesive coating of polysaccharides that stick to cell surface proteins including fibrinogen and fibronectin. Mature cells then colonize the layer of adherent cells on the new surface 
[16]. Biofilms sluff off materials termed endotoxins once they are mature. Endotoxins are important in implant failure.

Lipopolysaccharide (LPS) also called endotoxin, is an integral component of the outer cell membrane surrounding the inner cell wall of gram negative bacteria found in biofilms. It is associated with proteins and other cell membrane components, such as cell-wall anchored proteins (CWA) [23] [24]. LPS is the origin of endotoxins that are released during bacterial cell division and destruction of cell walls by antibiotics and host immune responses [24]. The adverse effects of biofilms are mediated by release of endotoxins at concentrations of less than 1 nanogram per $\mathrm{ml}$ [24]. The health effects of circulating endotoxins include fever, hypotension, septic shock, respiratory distress syndrome, disseminated intravascular coagulation, sepsis, periodontitis and aseptic loosening of orthopedic implants [24]. It has been reported that orthopedic, craniofacial and wrist implants can have substantial amounts of endotoxins remaining after sterilization [24] [25]. This can lead to aseptic loosening of implants [24] and prevents osteointegration of orthopedic implants [25].

Our results of the studies conducted with VOCT suggest that biofilms seen in pictures growing on Petri dishes in culture are too thin to be imaged by OCT; however, their resonant frequencies can be measured by VOCT. In wounds, where the tissue is easily imaged, it may be possible to get images of biofilms on the surface. Since the resonant frequencies of collagen in skin and that measured for biofilms are so different $(40 \mathrm{~Hz}$ for biofilms and about $100 \mathrm{~Hz}$ for collagen in skin), it should be possible to assess the presence of biofilms with VOCT.

\subsection{Viscoelastic Liquids}

Modern cataract surgery, utilizing phacoemulsification, is one of the most common surgeries performed in economically advanced countries. $3.5 \mathrm{M}$ procedures are performed in the United States alone. Viscoelastic solutions are an integral part of cataract surgery. These solutions are used during surgery to maintain normal intraocular spaces and pressure when the eye is opened and intraocular pressure is suddenly reduced to zero. These viscous solutions protect intraocular structures from irrigation damage and touch from intraocular instruments and devices and are used to delicately manipulate intraocular structures. These effects have been demonstrated in an animal models and humans during phacoemulsification [26].

Removal of a cataract during ophthalmic surgery is aimed at restoring visual acuity in order to secure a fast return to normal social life and work. To decrease postoperative endothelial cell loss, different ocular viscoelastic devices have been proposed to facilitate surgical tasks including maintaining space during surgery, and protecting the endothelial cells from trauma. These effects have been demonstrated in an animal model and humans during phacoemulsification [26].

Our results suggest that the cohesive behavior of viscoelastic solutions can be studied using VOCT; however, the behavior is more complicated than that observed for tissues and polymers. When studying the behavior of a drop of a 
viscous polymer solution with about $1 \%$ polymer content by weight, the behavior of water comes into play. While the polymer resonant frequency is much higher than that of water, the water appears to have at least one peak. The peak is at about $60 \mathrm{~Hz}$ and probably reflects cohesive energy of the water when placed on a glass slide. This peak disappears when a small amount of detergent is placed in the water suggesting that the surface tension of the water on glass is balanced internally within the droplet by cohesive energy forces between water molecules. When the resonant frequency of water is subtracted from that of the Provisc and Viscoat samples, there are two peaks seen for the two polymer solutions. While it is not possible to interpret the meaning of each of these peaks without studying the effects of different polymer solutions, it can be speculated that the first peak represents water polymer interactions while the second peak involves polymer-polymer interactions in solution. Further studies are needed at different pHs, concentrations, and polymer molecular weights to further characterize the significance of the two resonant frequencies seen for Provisc and Viscoat. Since both Provisc and Viscoat contain high molecular weight hyaluronic acid, future studies are needed that will look at this molecule in solution with varying molecular weights.

\section{Conclusion}

In this paper we report the use of VOCT to evaluate the viscoelastic behavior of tissues, polymeric materials, biofilms and viscoelastic solutions of macromolecules. Our results suggest that VOCT is a useful technique to characterize the behavior of cellular tissues and biofilms, polymeric implant materials and viscoelastic solutions used in medicine non-invasively and non-destructively. It is demonstrated that the modulus and resonant frequency squared per unit thickness is a characteristic feature that can be used to identify cellular and macromolecular materials in complex mixtures. Since synthetic polymers do not follow the same relationship between modulus and resonant frequency squared per unit sample thickness shown by cellular materials and collagenous tissues, further studies are needed to characterize additional polymeric materials to understand their behaviors.

\section{Acknowledgements}

The authors would like to thank Dr. Dale DeVore for helpful discussions concerning the properties of viscoelastic solutions.

\section{Conflicts of Interest}

The authors declare no conflicts of interest regarding the publication of this paper.

\section{References}

[1] Silver, F.H. and Shah, R. (2016) Measurement of Mechanical Properties of Natural 
and Engineered Implants. Advances in Tissue Engineering and Regenerative Medicine, 1, 1-9. https://doi.org/10.15406/atroa.2016.01.00004

[2] Shah, R., DeVore, D. and Pierce, M.G. (2016) Morphomechanics of Dermis-A Method for Non-Destructive Testing of Collagenous Tissues. Skin Research and Technology, 23, 399-406. https://doi.org/10.1111/srt.12349

[3] Shah, R., Pierce, M.C. and Silver, F.H. (2017) A Method for Non-Destructive Mechanical Testing of Tissues and Implants. Journal of Biomedical Materials Research Part A, 105, 15-22. https://doi.org/10.1002/jbm.a.35859

[4] Silver, F.H. and Shah, R.G. (2008) Mechanical Spectroscopy and Imaging of Skin Components in Vivo: Assignment of the Observed Moduli. Skin Research and Technology, 25, 47-53.

[5] Shah, R.G., DeVore, D. and Silver, F.H. (2018) Biomechanical Analysis of Decellularized Dermis and Skin: Initial in Vivo Observations Using OCT and Vibrational Analysis. Journal of Biomedical Materials Research Part A, 106, 1421-1427.

[6] Schuh, S., Kaestle, R., Sattler, E.J. and Welzel, J. (2016) Comparison of Different Optical Coherence Tomography Devices for Diagnosis of Non-Melanoma Skin Cancer. Skin Research and Technology, 22, 395-405.

https://doi.org/10.1111/srt.12277

[7] Baran, U., Choi, W.J. and Wang, R.K. (2016) Potential Use of OCT-Based Microangiography in Clinical Dermatology. Skin Research and Technology, 22, 238-246. https://doi.org/10.1111/srt.12255

[8] Robles Velasco, M.V.R., Simone Pfannemmuller de Abreu, S.R.P., Zanardi de Freitas, A., Bedin, V., Baby, A.R. and Miranda da Gama, R. (2016) Optical Coherence Tomography to Evaluate the Effects of Oxidative Hair Dye on the Fiber. Skin Research and Technology, 22, 430-434. https://doi.org/10.1111/srt.12283

[9] DeCarvalho, N., Schuh, S., Kindermann, N., Kästle, R., Holmes, J. and Welzel, J. (2018) Optical Coherence Tomography for margin definition of Basalcell Carcinoma before Micrographic Surgery-Recommendations Regarding the Marking and Scanning Technique. Skin Research and Technology, 24, 145-151.

https://doi.org/10.1111/srt.12407

[10] Odorici, G., Losi, A., Ciardo, S., Pellacani, G. and Conti, A. (2018) Non-Invasive Evaluation of Secukinumab Efficacy in Severe Plaque Psoriasis with Confocal Microscopy and Optical Tomography: A Case Report. Skin Research and Technology, 24, 160-162. https://doi.org/10.1111/srt.12409

[11] Sigsgaard, V., Themstrup, L., TheutRiis, P., Olsen, J. and Jemec, G.B. (2018) In Vivo Measurements of Blood Vessels' Distribution in Non-Melanoma Skin Cancer by Dynamic Optical Coherence Tomography-A New Quantitative Measure? Skin Research and Technology, 24, 123-128. https://doi.org/10.1111/srt.12399

[12] Kuck, M., Strese, H., Arash Alawi, S., Meinke, M.C., Fluhr, J.W., Burbach, G.J., Krah, M., Sterry, W. and Jurgen Lademann, J. (2014) Evaluation of Optical Coherence Tomography as a Non-Invasive Diagnostic tool in Cutaneous Wound Healing. Skin Research and Technology, 20, 1-7. https://doi.org/10.1111/srt.12077

[13] Mogensen, M., Nurnberg, B.M., Forman, J.L., Thomsen, J.B., Thrane, L. and Jemec, G.B.E. (2009) In Vivo Thickness Measurement of Basal Cell Carcinoma and Actinic Keratosis with Optical Coherence Tomography and 20-MHz Ultrasound. British Journal of Dermatology, 160, 1026-1033. https://doi.org/10.1111/j.1365-2133.2008.09003.x

[14] Gambichler, T., Regeniter, P., Bechara, F.G., Orlikov, A., Vasa, R., Moussa, G., Stucker, M., Altmeyer, P. and Hoffmann, K. (2007) Characterization of Benign and 
Malignant Melanocytic Skin Lesions Using Optical Coherence Tomography in $\mathrm{Vi}$ vo. Journal of the American Academy of Dermatology, 57, 629-637. https://doi.org/10.1016/j.jaad.2007.05.029

[15] Ring, H.C., Themstrup, L., Alette Banzhaf, C., Jemec, G.B.E. and Mogensen, M (2016) Dynamic Optical Coherence Tomography Capillaroscopya New Imaging Tool in Autoimmune Connective Tissue Disease. JAMA Dermatology, 152, 1142-1146. https://doi.org/10.1001/jamadermatol.2016.2027

[16] McCann, M.T., Gilmore, B.F. and Gorman, S.P. (2008) Staphylococcus Epidermidis Device Related Infections: Pathogenesis and Clinical Management. Journal of Pharmacy and Pharmacology, 60, 1551-1571. https://doi.org/10.1211/jpp.60.12.0001

[17] Percival, S.L., Vuotto, C., Donelli, G. and Lipsky, B.A. (2015) Biofilms and Wounds: An Identification Algorithm and Potential Treatment Options. Advances in Wound Care, 4, 389-397. https://doi.org/10.1089/wound.2014.0574

[18] Papagiannopoulos, G.A. and Hatzigeorgiou, G.D. (2011) On the Use of the Half-Power Bandwidth Method to Estimate Damping in Building Structures. Soil Dynamics and Earthquake Engineering, 31, 1075-1079. https://doi.org/10.1016/j.soildyn.2011.02.007

[19] Silver, F.H. (1994) Biomaterials, Medical Devices and Tissue Engineering: An Integrated Approach. Chapman and Hall, London. https://doi.org/10.1007/978-94-011-0735-8

[20] Romling, V. and Balasalobre, C. (2012) Biofilm Infections, Their Resistance to Therapy and Innovative Treatment Strategies. Journal of Internal Medicine, 272, 541-561. https://doi.org/10.1111/joim.12004

[21] Deasi, J.V., Mitchell, A.P. and Andes, D.R. (2014) Fungal Biofilms, Drug Resistance and Recurrent Infection. Cold Spring Harbor Perspectives in Medicine, 4, a019729.

[22] Gilbert, P., Das, J. and Foley, I. (1997) Biofilm Susceptibility to Antimicrobials. Advances in Dental Research, 11, 160-167. https://doi.org/10.1177/08959374970110010701

[23] Greenfield, E.M., Bi, Y., Ragab, A.A., Boldberg, V.M., Nalepka, J.L. and Seabold, J.M. (2005) Does Endotoxin Contribute to Aseptic Loosening of Orthopaedic Implants? Journal of Biomedical Materials Research, 72B, 179-185. https://doi.org/10.1002/jbm.b.30150

[24] Lieder, R., Peterson, P.H. and Sigurjonsson, O.E. (2013) Endotoxins the Invisible Companion in Biomaterials Research. Tissue Engineering, 19B, 391-402. https://doi.org/10.1089/ten.teb.2012.0636

[25] Bonsignore, L.A., Anderson, J.R., Lee, Z., Goldberg, V.M. and Greenfield, E.M. (2013) Adherent Lipopolysaccharide Inhibits Osteointegration of Orthopaedic Implants by Impairing Osteoblast Differentiation. Bone, 52, 93-101. https://doi.org/10.1016/j.bone.2012.09.011

[26] Storr-Paulsen, A., Norregaard, J.C., Farik, G. and Tarnhoj, J. (2007) The Influence of Viscoelastic Substances on the Corneal Endothelial Cell Population during Cataract Surgery: A Prospective Study. Acta Ophthalmologica Scandinavica, 85, 183-187. https://doi.org/10.1111/j.1600-0420.2006.00784.x 